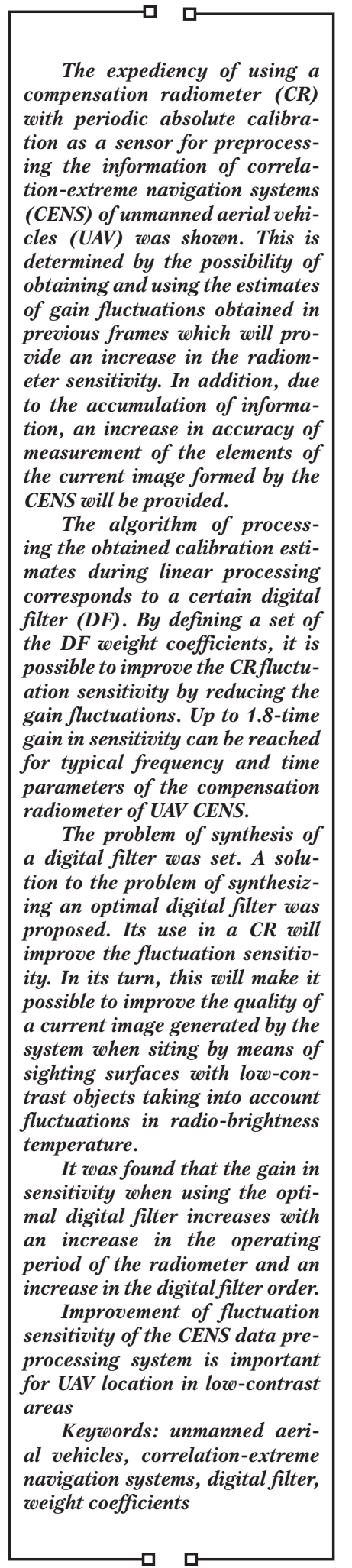

\title{
SYNTHESIS OF AN OPTIMAL DIGITAL FILTER OF A COMPENSATION RADIOMETER FOR RADIOMETRIC CORRELATION- EXTREME NAVIGATION SYSTEMS OF UNMANNED AERIAL VEHICLES
}

\author{
Nataliia Yeromina \\ $\mathrm{PhD}$, Senior Lecturer* \\ E-mail: ereminaport@gmail.com \\ Serhi i Petrov \\ $\mathrm{PhD}$, Associate Professor
}

Department of Physics, Electrical Engineering and Power Engineering

Ukrainian Engineering Pedagogics Academy

Universytetska str., 16, Kharkiv, Ukraine, 61003

Maks y m Volk

Doctor of Technical Sciences, Professor*

Olena Daki

Doctor of Technical Sciences, Associate Professor Department of Navigation and Operation of Technical Systems on Water Transport Danube Institute of Water Transport Fanagoriyska str., 7, Izmail, Ukraine, 68600

Volodymyr Cherednyk $\mathrm{PhD}$, Associate Professor

Department of Ship Power Plants, Auxiliary Mechanisms and Their Operation State University of Infrastructure and Technology

Kyrylivska str., 9, Kyiv, Ukraine, 04071

Iryna Zinchenko

Junior Researcher

Scientific Center of Information Protection

Military Institute of Telecommunications and Information Technologies named after Heroes of Kruty

Moskovs'ka str., 45/1, Kyiv, Ukraine, 01011

I hor Chernykh

$\mathrm{PhD}$, Associate Professor, Deputy Head of the Institute National Defence University of Ukraine Named After Ivan Cherniakhovskyi Povitroflotskyi ave., 28, Kyiv, Ukraine, 03049

Oleksiy Alekseienko

Scientific and Methodological Center of Scientific, Scientific and Technical Activities Organization**

Serhi i Mykus Doctor of Technical Sciences, Associate Professor, Head of Department Department of Information Technology and Information Security Employment Institute of the Troops (Forces) Support and Information Technologies** Volody m y r F r d yk Department of Combat Service Support** *Department of Electronic Computers Kharkiv National University of Radio Electronics Nauky ave., 14, Kharkiv, Ukraine, 61166 **National Defence University of Ukraine Named After Ivan Cherniakhovskyi Povitroflotskyi ave., 28, Kyiv, Ukraine, 03049

Received date 09.02.2021 Accepted date 12.04.2021 Published date
How to Cite: Yeromina, N., Petrov, S., Volk, M., Daki, O., Cherednyk, V., Zinchenko, I., Chernykh, I., Alekseienko, O., Mykus, S., Furdyk, V. (2021). Synthesis of the optimal digital filter of the compensation radiometer for radiometric correlation-extreme navigation system of unmanned flying machines. Eastern-European Journal of Enterprise Technologies, 2 (9 (110)), 79-86. doi: https://doi.org/10.15587/1729-4061.2021.230176

\section{Introduction}

Significant expansion of the scope of application of unmanned aerial vehicles (UAV) both in various sectors of the national economy and military affairs necessitates ensuring their effective functioning in various application conditions [1]. Modern high-precision UAV navigation systems are based on the application of strapdown inertial naviga- 
tion systems (SINS) which can be supplemented with both satellite navigation systems (SNS) and correlation-extreme navigation systems (CENS) [1]. However, despite the high accuracy indicators of SNS, their application for correction of UAV SINS is not always effective. This is connected with a significant dependence of their functioning on weather conditions and interference situations. In addition, the application of SNS leads to a loss of autonomy of the UAV navigation system.

CENS including optoelectronic CENS as the best systems in terms of accuracy have no these shortcomings [2]. However, the efficiency of these systems also depends on meteorological conditions and interference situations. In such conditions, significant worsening of quality of generated current images (CI) used in CENS to solve the UAV positioning problem can occur. Based on this, a millimeter-wave radiometric channel with a sufficiently high resolution can be used as an additional information retrieval channel. However, along with this advantage, the positioning accuracy of the UAV with radiometric CENS may turn out to be insufficient because of the low sensitivity of the radiometer. The problem of improving the sensitivity of the radiometric sensor becomes even more obvious when using reference areas with low-contrast objects for UAV positioning. Thus, ensuring the required efficiency of UAV application necessitates the search for ways to increase the fluctuation sensitivity of the radiometers used in CENS.

The influence of seasonal and diurnal variation of radio-brightness temperature of the sighting surface objects further emphasizes the relevance of studies in the field of further improvement of radiometric channels for receiving and preprocessing information signals.

\section{Literature review and problem statement}

Models of radiometric CI and reference images (RI) are given in [1]. The possibility of reducing the influence of geometric distortions on the accuracy of CENS positioning was shown. Algorithms of forming RI and decision function were proposed. The problem of developing a method of forming the CENS decision function was solved. The method is based on the current image preprocessing which consists of its stratification relative to the average value of the background radio brightness temperature and construction of a set of selective images. At the same time, the authors ignored the issues of improving the quality of the TI being generated.

The results of the development of a method of localization of the siting object in the TI in the event of the appearance of false objects caused by geometric distortions are presented in [2]. Sizes of the siting objects and the degree of contrast between them were not taken into account.

The results of localization of object contours in images based on the Hough transformation are presented in [3], however, the potential influence of CI distortions on the problem-solution was not considered.

Study [4] has suggested localizing objects in images based on fractal analysis. The influence of distortions and blurring of boundaries between objects in images were not taken into account.

It was proposed in [5] to sharpen the outer contours of recognition objects by means of detecting contour points of the objects. The CI distortions and the ways to eliminate them have not been studied.
The solution to the problem of localizing objects in images for a three-dimensional ground scene is given in [6] without taking into account its influence on perspective CI distortions.

A CENS diagram with an operational mapping unit is presented in [7]. The unit implements the well-known approach to simultaneous positioning and mapping based on recursive Bayesian estimation. However, CI formation by means of comparing with EI based on mapping information is not shown.

Methods of image segmentation using various informative parameters are considered in [8]. The possibility of forming homogeneous regions for finding additional information about the region of interest in the image is shown. The methods are generalized and do not take into account the peculiarities of CENS functioning.

Results of applying an L-band radiometer on a UAV are presented in [9]. Experimental studies indicate the presence of essential errors in positioning.

Results of applying inexpensive radiometric hyperspectrometers are presented in [10]. It was established that planimetric accuracy can reach $4.6 \mathrm{~m}$ at a height of $344 \mathrm{~m}$ above ground which shows that the application of radiometers in navigation is promising.

A method of efficient synthesis of RI based on fractal analysis is proposed in [11]. The method was developed without taking into account potential distortions in original images.

It is proposed in [12] to form RI based on the construction of a field of correlation analysis. The influence of distorting factors and the magnitude of contrast between objects were not considered.

A method was proposed in [13] for improving the accuracy of CENS siting in the presence of several bright objects on a sighting surface and deliberate interference. The problem of siting was solved in the presence of a group of bright objects on the sighting surface. Brightness variation was not taken into account.

Approaches to detection and localization of objects in images are based in [14] on the Hough transform. The problem is solved without taking into account the influence of distorting factors on CI.

The results obtained in developing methods of secondary image processing based on adaptation to quantization value are presented in [15].

Results of comparative analysis of models of fluctuation sensitivity of radiometers of various types are presented in [16]. The preference of using a compensation radiometer $(\mathrm{CR})$ in comparison with a modulation radiometer is shown when studying noise signals close to absolute zero. No ways of improvement of the fluctuation sensitivity are given.

The results of studies on improving the quality of primary radiometric images formed by means of radiometric scanning devices of compensation type using weight processing of amplitude-phase field distribution in antenna aperture using window functions (classical and Kravchenko functions) are presented in [17]. An increase in fluctuation sensitivity of the radiometer was not considered.

The results of the development of an algorithm of optimal processing the signals of intrinsic radio-thermal radiation in a CR with an unstable gain are presented in [18]. The development has provided low sensitivity to fluctuations in the pre-detector section of the radiometer.

Thus, the solution to the problem of forming images by radiometric channel using low-contrast objects and taking 
into account fluctuations of brightness temperature has not received proper development. It is explained by the fact that more attention was paid to the methods and algorithms of secondary processing. The issues of improving the primary processing systems were assumed as already resolved and were considered without taking into account peculiarities of the algorithms of functioning of secondary processing systems. In addition, the study of ways to improve the efficiency of CENS functioning was performed for high-speed aircraft. At the same time, it was not taken into account that the range of UAV velocities allows the use of the frame-by-frame mode of operation of the CENS radiometric channel. These circumstances indicate the advisability of using a CR with periodic absolute calibration which has the highest potential sensitivity in preprocessing channels. Taking into account the adaptive methods of secondary information processing in CENS developed to date, it is proposed to use estimates of the gain coefficient (GC) obtained in previous frames. Due to the accumulation of information, this will make it possible to ensure an increase in accuracy of measuring the image elements. If linear processing of calibration samples obtained in the previous frames is used, then such an algorithm will correspond to the algorithm of some digital filter (DF).

Application of radiometers using non-recursive DF in the calibration signal channel in space systems of earth survey is known [19]. In this case, the radiometer structure was analyzed for the case of equal weighting coefficients of the $\mathrm{DF}$. That is, the average value of the calibration readings obtained in the previous frames of the system operation was calculated and the concept of the DF was not used at all. Obviously, it is necessary to apply the weighted mean value of calibration counts. The closer in time the calibration count is to the moment of taking the frame, the greater the corresponding weight coefficient of the DF should be. In this regard, it becomes necessary to solve the problem of synthesizing the optimal DF at which the sensitivity of the $\mathrm{CR}$ will be maximum. This will improve the quality of images of the sighting surfaces with low-contrast objects formed by the system.

\section{The aim and objectives of the study}

The study objective implies improving the fluctuation sensitivity of the CENS radiometric channel for UAV positioning in terrain areas with low-contrast objects.

To achieve this objective, it is necessary to solve the following tasks:

- form statement of the problem of synthesizing the optimal DF of the UAV CENS CR;

- solve the problem of synthesizing the optimal DF of the $\mathrm{CR}$;

- perform numerical calculations and analyze the results of synthesis of optimal DF.

\section{Materials and methods used in the study}

A CR with periodic absolute calibration was chosen as a system of preprocessing the CENS radiometric channel. The choice in favor of this radiometer was determined by the simplicity of its circuit design and dependence of its transmission coefficient on noise parameters of reference generators and values of the output digital function. To ensure measurement of estimates in absolute scale in each period, it was necessary to measure signals of two reference sources with equivalent noise temperatures $\mathrm{T}_{\mathrm{e} 1}$ and $\mathrm{T}_{\mathrm{e} 2}$, for example, $\mathrm{T}_{\mathrm{e} 1}=\mathrm{T}_{\max }, \mathrm{T}_{\mathrm{e} 2}=\mathrm{T}_{\min }$. The receiver transfer characteristic was assumed to be linear. The optimal digital filter was sought in the class of linear non-recursive digital filters for which there is no problem of stability. The dynamic range of such a radiometer is from $T_{\min }$ to $\mathrm{T}_{\max }$.

Statement of the problem of synthesis of the optimal digital filter of a CR with periodic absolute calibration was formed using the method of synthesis of non-recursive digital filters. When setting the problem, it was taken into account that the UAV CENS operates in real time. This means that it was sufficient to synthesize a digital filter with no memory.

Description of measurement readings, estimates of antenna temperature, errors in determining the coefficient of transmission of the receiving path are based on the methods of the theory of signal parameter estimates and radiometry.

The amplitude-frequency characteristic of the digital filter was determined based on the z-transformation from the impulse transient response. The transfer function was determined using the theory of integrals and series.

It was proposed to synthesize the optimal digital filter according to the criterion of minimum dispersion of the estimate of the radiometer antenna temperature which is ensured by determining the set of its corresponding weight coefficients. The set of weighting coefficients of the digital filter which provides for minimum dispersion of estimate of the radiometer antenna temperature was determined using the Lagrange multiplier method. In this case, the condition of ensuring stationarity of the Lagrange function in terms of weight coefficients was imposed. The Lagrange multipliers at the point of local extremum were defined using the matrix theory.

It is advisable to analyze the results of the synthesis of the digital filter on the basis of an estimate of the gain in sensitivity of the CR for real frequency parameters of the radiometer and temporal conditions for the formation of the frame of images of the sighting surface of the UAV CENS. The parameters for calculation were taken from [19]. The following radiometer parameters were used in calculations: $\Delta \mathrm{f}=1.0 \mathrm{GHz} ; \mathrm{A}=0.65^{*} 10^{-10}$; for $\mathrm{J}=2$ and $\mathrm{J}=10 ; \mathrm{t}_{\mathrm{k}}=(0.2 \ldots 100) \mathrm{s}$, the ratio $t_{\mathrm{s}} \mid t_{\mathrm{k}}$ changed from 0 to 1 .

Numerical estimates of sensitivity gain when using optimal DFs were obtained on the basis of numerical simulation. The initial data for modeling corresponded to real time conditions for the formation of the CENS of image frames and the frequency parameters of the radiometer corresponded to the typical values.

\section{The results of synthesis of a digital filter in the reference channel of a $C R$ with periodic absolute calibration}

\subsection{Statement of the problem of synthesis of an optimal digital filter of a CR with periodic absolute cal- ibration}

Fig. 1 shows a block diagram of a CR with periodic absolute calibration and the principle of its operation is explained by the pulse-time diagram shown in Fig. 2 .

It is of interest to solve the problem of synthesizing the optimal DF, i.e., determine such a set of weight coefficients at which the sensitivity of the radiometer is maximal. 


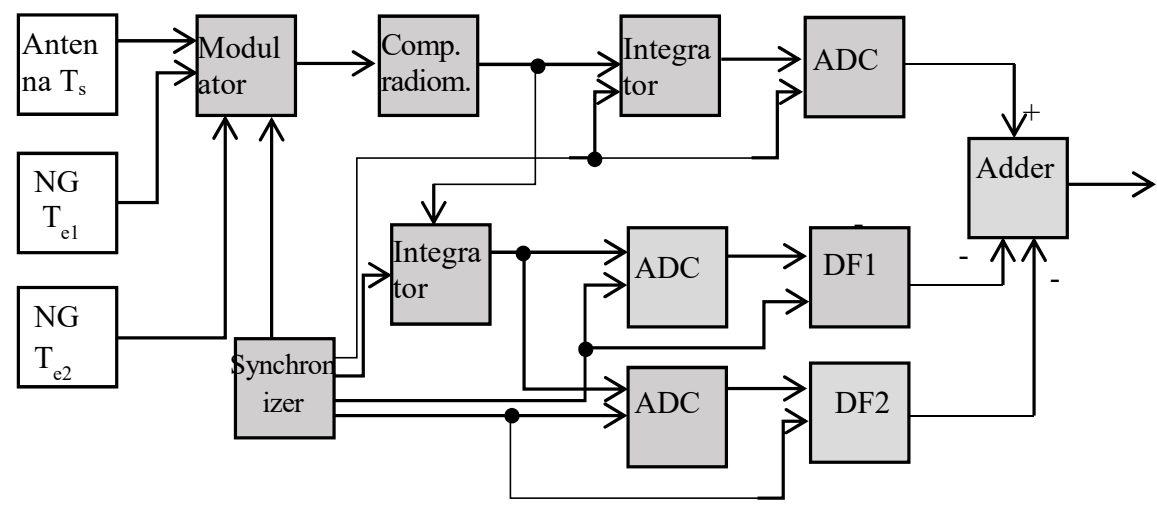

Fig. 1. Block diagram of a $\mathrm{CR}$ with periodic absolute calibration

digital function used to form one value of the output digital function.

Note that, DFs with memory $(L>0)$ can be used in the systems of processing accumulated data, in particular in earth survey systems in which a mode of operation in real time is not required. Only digital filters with $L=0$ can be used in systems of the class under consideration.

The following can be written for each measurement count:

$$
\begin{aligned}
& \bar{u}_{n}\left(t_{s}\right)=\frac{1}{\tau} \int_{n t_{k}+t_{s}-\tau / 2}^{n t_{k}+t_{s}+\tau / 2} v(t) \mathrm{d} t, \\
& 3 t_{k} / 2<t_{s}<t_{k}-\tau_{k} / 2,
\end{aligned}
$$

integrator) at each cycle with a period $t_{k}$ is connected either to the antenna or to the output of one of the reference noise generators. These generators are characterized by noise temperatures $T_{\mathrm{e} 1}$ and $\mathrm{T}_{\mathrm{e} 2}$. The modulation switch is controlled by a synchronizer.

In this case, a series of measuring counts of the radiometer output voltage are formed with the help of the integrator. They are converted into a digital code by means of an analog-to-digital converter (ADC). The integrator is characterized by the integration time $\tau$.

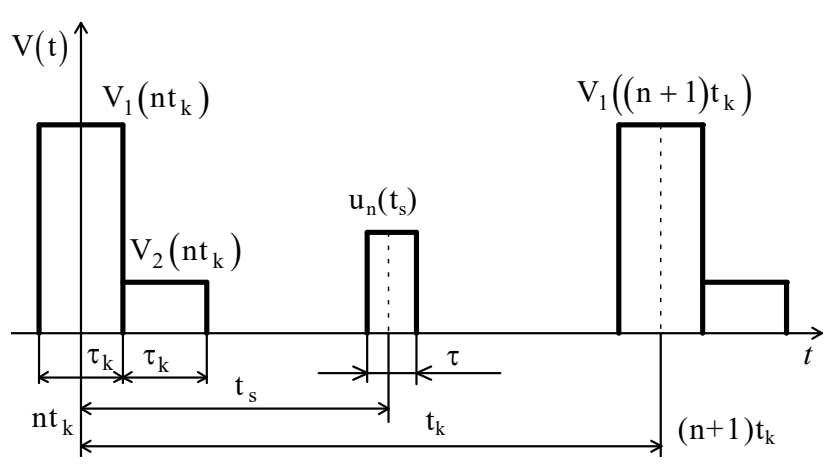

Fig. 2. Pulse-time diagram describing the principle of operation of the $\mathrm{CR}$ with periodic absolute calibration

In this case, a pair of calibration counts are formed using an integrator with integration time $\tau_{k}$ :

$$
\begin{aligned}
& \bar{v}_{1}\left(n t_{k}\right)=\frac{1}{\tau_{k}} \int_{n t_{k}-\tau_{k} / 2}^{n t_{k}+\tau_{k} / 2} v(t) \mathrm{d} t, \\
& \bar{v}_{2}\left(n t_{k}\right)=\frac{1}{\tau_{k}} \int_{n t_{k}+\tau_{k} / 2}^{n t_{k}+3 \tau_{k} / 2} v(t) \mathrm{d} t .
\end{aligned}
$$

Let us restrict ourselves to the search for optimal DF in a linear non-recursive DF class for which there is no stability problem and the algorithm is determined by the expression [20-22]:

$$
V_{j}\left(n t_{k}\right)=\sum_{i=-L}^{J} h_{i} \bar{v}_{j}\left[(n-i) t_{k}\right], \quad j \in \overline{1,2}
$$

where $h_{i}$ is the DF weight coefficients; $L, J=0,1, \ldots, Q=L+J+1$ is the DF order, that is, the number of counts of the input

belonging to the $\mathrm{n}$-th cycle and delayed by time $\mathrm{t}_{\mathrm{s}}$ relative to the moment $\mathrm{nt}_{\mathrm{k}}$; the antenna temperature estimate is determined from expression [23]:

$$
\begin{aligned}
& \hat{T}_{s n}\left(t_{s}\right)=\beta\left\{\left[\bar{u}_{n}\left(t_{s}\right)-V_{1}\left(n t_{k}\right)\right] c\left(n t_{k}\right)+T_{e 1}\right\}+ \\
& +(1-\beta)\left\{\left[\bar{u}_{n}\left(t_{s}\right)-V_{2}\left(n t_{k}\right)\right] c\left(n t_{k}\right)+T_{e 2}\right\},
\end{aligned}
$$

where

$$
\begin{aligned}
& \beta=\frac{T_{s}-T_{e 2}}{T_{e 1}-T_{e 2}} \in[0,1], \\
& c\left(n t_{k}\right)=\frac{T_{e 1}-T_{e 2}}{V_{1}\left(n t_{k}\right)-V_{2}\left(n t_{k}\right)},
\end{aligned}
$$

$c\left(n t_{k}\right)$ is the transmission coefficient of the receiver.

Introduce centered random quantities:

$$
\delta V_{j}\left(n t_{k}\right)=V_{j}\left(n t_{k}\right)-M V_{j}\left(n t_{k}\right), \quad j \in \overline{1,2}
$$

and represent the relation (5) in the form:

$$
c\left(n t_{k}\right)=c_{0} /(1+\delta V / \Delta V),
$$

where

$$
\begin{aligned}
& \Delta V=M V_{1}-M V_{2}, \quad c_{0}=\left(T_{s 1}-T_{s 2}\right) / \Delta V, \\
& \delta V=\delta V_{1}-\delta V_{2} .
\end{aligned}
$$

Assume $\delta V<<\Delta V$ and obtain:

$$
c\left(n t_{k}\right) \approx c_{0}(1-\delta V / \Delta V) .
$$

Then the relative root-mean-square error $\varepsilon$ in determining the coefficient $c\left(n t_{k}\right)$ from formula (5) is:

$$
\varepsilon=\frac{\sqrt{M\left(c-c_{0}\right)^{2}}}{c_{0}}=\frac{\sqrt{\left(\sigma_{T e 1}^{2}+\sigma_{T e 2}^{2}\right)}}{T_{e 1}-T_{e 2}}<\frac{\sqrt{2} \sigma_{T e 1}}{T_{e 1}-T_{e 2}} .
$$

In practical applications, the radiometer sensitivity is significantly less than its dynamic range, so we will consider $c\left(n t_{k}\right) \approx \mathrm{c}_{0} \forall n \in N$. Then, after performing statistical averag- 
ing over the ensemble of realizations in (5), the following is obtained:

$$
M \hat{T}_{s}=T_{s}+\left(1-\sum_{i=-L}^{J} h_{i}\right)\left[T_{n}+\beta T_{e 1}+(1-\beta) T_{e 2}\right],
$$

whence it follows that in order for the estimate $\hat{T}_{s}$ be unbiased, i. e., $M \hat{T}_{s}-T_{s}=0$, the DF weight coefficients must satisfy the following condition:

$$
\sum_{i=-L}^{J} h_{i}=1
$$

It can be shown that for the estimated $\hat{T}_{s}$ variance

$$
\sigma_{T_{s}}^{2}\left(t_{s}\right)=c_{0}^{2} M\left[\bar{u}_{n}\left(t_{s}\right)-\beta V_{1}\left(n t_{k}\right)-(1-\beta) V_{2}\left(n t_{k}\right)\right]^{2},
$$

the following ratio is true:

$$
\max _{\left.T_{s} \in T_{\left.T_{2}, T_{e}\right]}\right]} \sigma_{T_{s}}^{2}\left(t_{s}\right)=\sigma_{T_{e 1}}^{2}\left(t_{s}\right)=c_{0}^{2} M\left[\bar{u}_{n}\left(t_{s}\right)-V_{1}\left(n t_{k}\right)\right]^{2}
$$

i. e., the variance is maximum at $T_{s}=T_{e 1}$ (in this case, $\beta=1$ ). Therefore, in what follows, we restrict ourselves to calculating the radiometer sensitivity for this worst case.

Transform expression (8) to the following form:

$$
\sigma_{T_{s}}^{2}\left(t_{s}\right)=c_{0}^{2} \int_{-\infty}^{\infty} S_{v}(f)|H(f)|^{2} \mathrm{~d} f
$$

where $S_{V}(f)$ is the spectral voltage density at the radiometer output which for the case $q=T_{s} / T_{n}<<1$ is determined by the ratio:

$$
\begin{aligned}
& S_{v}(f)=\frac{T_{n}^{2}}{c_{0}^{2}}\left[S_{g}(f)+\frac{1}{\Delta f}\right] ; \\
& H(f)=\sin c(\pi f \tau) e^{-j 2 \pi f t_{s}}-\sin c\left(\pi f \tau_{k}\right) H_{d f}(f) .
\end{aligned}
$$

The frequency response (FR) $H_{d f}(f)$ of the digital filter is determined by z-transformation from the impulse transient response [20]:

$$
H_{d f}(f)=\sum_{i=-L}^{J} h_{i} e^{-j 2 i \pi f t_{k}}
$$

Then the transfer function $|H(f)|^{2}$ takes the form:

$$
\begin{aligned}
& |H(f)|^{2}=\left[\begin{array}{l}
\sin c \pi f \tau \cos 2 \pi f t_{s}- \\
-\sin c \pi f \tau_{k} \sum_{i=-L}^{J} h_{i} \cos 2 \pi f i t_{k}
\end{array}\right]^{2}+ \\
& +\left[\sin c \pi f \tau \cos 2 \pi f t_{s}-\sin c \pi f \tau_{k} \sum_{i=-L}^{J} h_{i} \sin 2 \pi f i t_{k}\right]^{2} .
\end{aligned}
$$

This expression can be converted to the following form:

$$
\begin{aligned}
& |H(f)|^{2}=\sin c^{2} \pi f \tau+\sin c^{2} \pi f \tau_{k} \times \\
& \times \sum_{i, l=-L}^{J} h_{i} h_{l} \cos 2 \pi f t_{k}(l-i)- \\
& -2 \sin c \pi f \tau \sin c \pi f \tau_{k} \sum_{i=-L}^{J} h_{i} \cos 2 \pi f\left(i t_{k}+t_{s}\right) .
\end{aligned}
$$

Calculate the integral

$I=\int_{0}^{\infty}|H(f)|^{2} d f$

Using tabular integrals [24]

$\int_{0}^{\infty} \sin c^{2} a x \mathrm{~d} x=a \pi / 2, a>0$

$\int_{0}^{\infty} \sin c^{2} a x \cos 2 b x \mathrm{~d} x=\left\{\begin{array}{l}\pi(a-b) / 2, b<a ; \\ 0, b>a\end{array}\right.$

the following is obtained:

$$
\begin{aligned}
& \int_{0}^{\infty} \sin c^{2} \pi f \tau \mathrm{d} f=1 / 2 \tau ; \\
& \int_{0}^{\infty} \sin c^{2} \pi f \tau_{k} \cos \left[2 \pi f t_{s}(i-k)\right] \mathrm{d} f=\frac{1}{2 \tau_{k}} \delta_{i k} ; \\
& \int_{0}^{\infty} \sin c^{2} \pi f \tau \sin c^{2} \pi f \tau_{k} \cos \left[2 \pi f\left(k t_{k}-t_{s}\right)\right] \mathrm{d} f=0,
\end{aligned}
$$

where it is taken into account that $t_{s}>\tau, \tau_{k}$.

Now expression (9) can be represented as:

$$
\sigma_{T_{s}}^{2}(\mathbf{h})=\left(\delta T_{k}\right)^{2}\left[1+\frac{\tau}{\tau_{k}} \sum_{i=-L}^{J} h_{i}^{2}+A \Delta f \tau \int_{-\infty}^{\infty} \frac{|H(f)|^{2}}{f^{\gamma}} \mathrm{d} f\right],
$$

where $\delta T_{k}$ is the fluctuation sensitivity of the CR;

$$
\mathbf{h}=\left(h_{-L}, \ldots, h_{J}\right) \in \mathbf{R}^{L+J+1} .
$$

Integration in (12) gives the following:

$$
\sigma_{T_{s}}^{2}(\mathbf{h})=\left(\delta T_{k}\right)^{2}[1+\Delta f \tau F(\mathbf{h})],
$$

where

$$
\begin{aligned}
& F(\mathbf{h})=D\left(\tau, \tau_{k}\right)+\sum_{i=-L}^{J} h_{i}\left[\begin{array}{l}
\frac{h_{i}}{\Delta f \tau_{k}}+2 C\left(\tau, i t_{k}+t_{s}\right)- \\
-\sum_{l=-L}^{J} h_{l} C\left(\tau_{k},|l-i| t_{k}\right)
\end{array}\right], \\
& D(x, y)=2 A \int_{0}^{\infty}(\sin c \pi f x-\sin c \pi f y)^{2} \mathrm{~d} f / f^{\gamma}, \\
& C(x, y)=4 A \int_{0}^{\infty} \sin c \pi f x \sin c \pi f \tau_{k} \sin ^{2} \pi f y \mathrm{df} / f^{\gamma} .
\end{aligned}
$$

As a result of calculating the integrals (15), (16), the following is obtained:

$$
\begin{gathered}
C(x, y)=\frac{A \Gamma(-\gamma) \cos \frac{\gamma+1}{2} \pi}{2 \pi^{2} x \tau_{k}(\gamma+1)}(2 \pi y)^{\gamma+1} \times \\
\times\left[\begin{array}{l}
\left(1+\frac{\tau_{k}-x}{2 y}\right)^{\gamma+1}-\left(1+\frac{\tau_{k}+x}{2 y}\right)^{\gamma+1}+ \\
+\left(1-\frac{\tau_{k}-x}{2 y}\right)^{\gamma+1}-\left(1-\frac{\tau_{k}+x}{2 y}\right)^{\gamma+1}
\end{array}\right],
\end{gathered}
$$




$$
\begin{aligned}
& D(x, y)=\frac{2 A \cos \frac{\gamma+1}{2} \pi \Gamma(-\gamma) \pi^{\gamma-1}}{x y(\gamma+1)} \times \\
& \times\left[(y-x)^{\gamma+1}-(y+x)^{\gamma+1}+y(2 x)^{\gamma}+x(2 y)^{\gamma}\right] .
\end{aligned}
$$

In the case of $y>>x, \tau_{k}$, formula (17) can be simplified by expanding each of the terms in square brackets into a Taylor series and keeping the quadratic terms. As a result, the following is obtained:

$$
C(x, y) \approx-2 A \gamma(2 \pi y)^{\gamma-1} \cos \frac{\gamma+1}{2} \pi \Gamma(-\gamma) \text {. }
$$

Now we can formulate the problem of synthesizing a DF that is optimal according to the criterion of minimum variance of the estimate $\hat{T}_{s}$, determined from formula (13): find a set of weight coefficients of the DF, i. e., the vector $\hat{\mathbf{h}}=\left(\hat{h}_{-L}, \ldots, \hat{h}_{J}\right) \in \mathbf{R}^{L+J+1}$, minimizing the function $F(\mathbf{h})$ given by relation (14) subject to constraint (7).

\section{2. Synthesis of an optimal digital filter of a CR with} periodic absolute calibration

The problem posed in p. 5.1 belongs to the class of smooth extremal problems with a constraint of equality type [25]. To solve it, set up the Lagrange function:

$$
L(\mathbf{h}, \lambda)=F(\mathbf{h})+\lambda\left(\sum_{i=-L}^{J} h_{i}-1\right),
$$

and use the theorem [25]:

"If $\hat{\mathbf{h}}$ is a local extremum point in the problem (14), (17), then there is such Lagrange multiplier $\hat{\lambda}$ that the condition of the Lagrange function stationarity is satisfied with respect to h", i. e.,

$$
\frac{\partial L}{\partial h_{i}}(\hat{\mathbf{h}}, \hat{\lambda})=0, \quad i \in \overline{-L, J}
$$

To find the optimal element $(\hat{\mathbf{h}}, \hat{\lambda})$, take a system of $L+J+2$ equations:

$$
\left\{\begin{array}{l}
2\left\{\begin{array}{l}
\frac{\hat{h}_{i}}{\Delta f \tau_{k}}+C\left(\tau, i t_{k}+t_{s}\right)- \\
-\sum_{l=-L}^{J} \hat{h}_{l} C\left[\tau_{k},|l-i| t_{k}\right]
\end{array}\right\}+\hat{\lambda}=0, i \in \overline{-L, J} ; \\
\sum_{l=-L}^{J} \hat{h}_{l}=1 .
\end{array}\right.
$$

Let us restrict ourselves to the class of physically realizable DFs with $L=0$. Write the system (21) in a matrix form:

$$
\mathbf{y}=\mathbf{Z} \mathbf{x}
$$

where

$$
Z=\left[\begin{array}{cccccc}
\beta & -C_{1} & -C_{2} & \cdots & -C_{J} & 1 / 2 \\
-C_{1} & \beta & -C_{1} & \cdots & -C_{J-1} & 1 / 2 \\
-C_{2} & -C_{1} & \beta & \cdots & -C_{J-2} & 1 / 2 \\
\cdots & \cdots & \cdots & \cdots & \cdots & \cdots \\
-C_{J} & -C_{J-1} & -C_{J-2} & \cdots & \beta & 1 / 2 \\
1 & 1 & 1 & \cdots & 1 & 0
\end{array}\right],
$$

$$
\begin{aligned}
& y=\left[-C\left(\tau, t_{s}\right),-C\left(\tau, t_{k}+t_{s}\right) \ldots,-C\left(\tau, J t_{k}+t_{s}\right), 1\right]^{\prime} \in R^{J+2} \\
& x=\left[h_{0}, \ldots, h_{J}, \lambda\right]^{\prime} \in R^{J+2} \\
& C_{i}=C\left(\tau_{k}, i \tau\right), \quad i \in \overline{1, J}, \quad \beta=1 /\left(\Delta f \tau_{k}\right)-C\left(\tau_{k}, 0\right)
\end{aligned}
$$

The system of equations (24) can be solved by one of the known methods. When constructing the system matrix, it is expedient to assume that the submatrix corresponding to the variables $h_{0}, \ldots, h_{J}$ is symmetric.

The following is obtained from (13) for the coefficient $K=\sigma_{T s} / \delta T_{k}$ :

$$
K=\sqrt{1+\Delta f \tau F} .
$$

Note that solution to system (21) takes the following form for $A=0$ :

$$
h_{i}=(J+1)^{-1}, \quad(i \in \overline{0, J})
$$

Consequently, in this case, the DF with the same weight coefficients is optimal. Call such digital filter a quasi-optimal filter.

5. 3. Analysis of the results of synthesis of a digital filter and elaboration of recommendations on their practical use

Fig. 3 shows the results of calculating the coefficient $K$ depending on the ratio $t_{s} / t_{k}$ for optimal DFs with $J=2$ (Fig. $3, a$ ) and $J=10$ (Fig. $3, b$ ) (thick curves) and corresponding quasi-optimal DFs (thin curves).
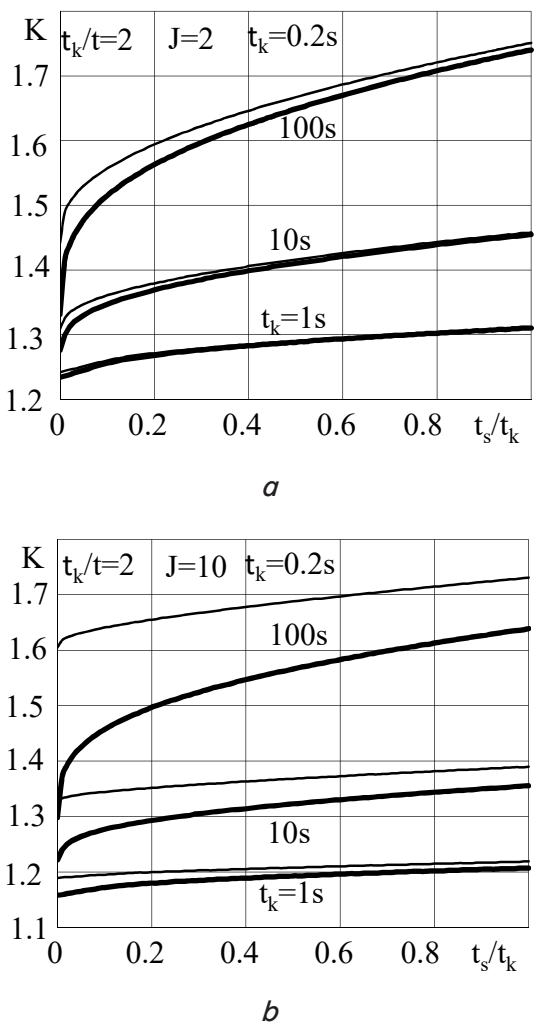

Fig. 3. Graph of $K\left(t_{s} / t_{k}\right)$ dependence: $a-$ at $J=2 ; b-$ at $J=10$

The following radiometer parameters were used in calculations: $\Delta f=1.0 \mathrm{GHz} ; A=0.65 \cdot 10^{-10} ; \gamma=1.3$. The calculation 
results indicate that the sensitivity gain when using optimal DFs increases with an increase in the period of the radiometer operation (the period between frames) $t_{\mathrm{k}}$ and an increase in the DF order $J$. For systems of the considered class $t_{k} \approx 1 \mathrm{~s}$, the gain turns out to be insignificant when using optimal DFs and it is possible to use quasi-optimal digital functions with the same weight coefficients to increase the system speed.

Fig. 4 illustrates the dependence of the coefficient $K$ on the DF order $J$. For optimal DFs, gain in sensitivity increases monotonically with an increase in $J$ and for quasi-optimal DFs, there is an optimal value of $J$. With an increase in the period $\tau$, the optimum shifts to the region of smaller values of $J$.

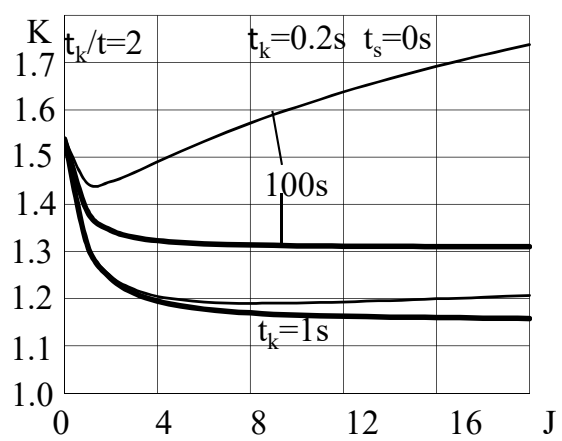

Fig. 4. Dependence of $K$ on $J$

It follows from Fig. 4 that in systems of the class under consideration characterized by the value of $t_{k} \approx 1 \mathrm{~s}$, the use of a DF of the order of $J>5$ practically does not give a gain in sensitivity leading to an increase in computational costs. Therefore, it is advisable to use the DF with $J \leq 5$ to solve the set tasks.

\section{Discussion of the results obtained in synthesis of the digital filter and development of recommendations for their practical application}

The presented results are a continuation of the development of methods for increasing the efficiency of the UAV CENS functioning. The proposed approach features further improvement of the CENS data preprocessing system through improvement of the radiometer's fluctuation sensitivity. This problem is solved by determining the set of weight coefficients of DF of CR with periodic absolute calibration at which the gain fluctuations will be minimal.

During the synthesis of the digital filter of the CR, a number of initial relations (9), (12) were obtained for the variance of estimation of the radiometer antenna temperature. Based on these relations, expression (13) was obtained for the variance of the antenna temperature estimate as a function of the DF weight coefficients. This has made it possible to formulate the problem of finding a set of the filter weight coefficients from the criterion of minimum variance of the estimate determined from formula (13) minimizing the function $F(h)$ given by relation (14). Since the expression (4) for the radiometer antenna temperature includes the transmission coefficient of the receiving path (5), a connection between fluctuations of the $\mathrm{CR}$ amplification coefficient and the DF weight coefficients becomes obvious. In other words, the set of GF weight coefficients at which gain fluctuations are minimal provides the maximum fluctuation sensitivity of the radiometer.

In the course of solving the problem of synthesizing a DF, it was found that a digital filter with the same weight coefficients is optimal (26). Numerical calculations have shown that when using the UAV CENS, the gain in sensitivity from the use of optimal DF turns out to be insignificant in comparison with the quasi-optimal DF (with the same weight coefficients). Therefore, it is advisable to use quasi-optimal digital filters. In this case, the use of digital filters of order $J>5$ is virtually inexpedient.

The advantage of the considered approach consists in an increase in fluctuation sensitivity of the CR based on the use of digital filters with the same weight coefficients up to potentially achievable sensitivity. This ensures the simplicity of the signal processing device. In addition, such radiometers are simple in their circuitry, e.g., in comparison with the two-channel CR. They have small dimensions which makes it possible to use them in the UAV CENS.

Thus, the use of CR with periodic absolute calibration in combination with adaptive algorithms of the secondary image processing systems will provide a solution to the problem of UAV positioning in real time when using low-contrast objects.

It is advisable to carry out further development of this study in two lines. The first line concerns defining the object types which, by their contrast, can be used for UAV positioning taking into account fluctuations of the radio-brightness temperature. The second line concerns the coordination of functioning of the considered system for preprocessing information of radiometric UAV CENS with a secondary processing system based on adaptive algorithms.

In the aggregate, the study results will be useful in improving the software package for the formation of a unimodal decision function in various conditions of radiometric CENS operation.

\section{Conclusions}

1. Statement of the problem of synthesis of DFs has been worked out. The problem statement differs from the known one in that determination of the set of the DF weighting coefficients is made according to the criterion of minimum variance of the radiometer antenna temperature.

2. The problem of synthesis of optimal DF was solved. It was found that in the absence of fluctuations in the gain of the receiving path, the DF with the same weight coefficients is optimal. In this case, such a DF is quasi-optimal.

3. Numerical calculations showed that gain in sensitivity monotonically increases with an increase in $J$ for optimal DF and there is an optimal value of $J$ for quasi-optimal DF. It was found that with an increase in the period $\tau$, the optimum shifts to the region of smaller values of $J$. In the UAV CENS featuring the value of $t_{k} \approx 1 \mathrm{~s}$, the use of a DF of the order of $J>5$ virtually does not ensure a gain in sensitivity but is leading to higher computational costs. Therefore, it is advisable to use the DF with $J \leq 5$ to solve the set tasks. 


\section{References}

1. Sotnikov, O., Kartashov, V. G., Tymochko, O., Sergiyenko, O., Tyrsa, V., Mercorelli, P., Flores-Fuentes, W. (2019). Methods for Ensuring the Accuracy of Radiometric and Optoelectronic Navigation Systems of Flying Robots in a Developed Infrastructure. Machine Vision and Navigation, 537-577. doi: https://doi.org/10.1007/978-3-030-22587-2_16

2. Sotnikov, A., Tarshyn, V., Yeromina, N., Petrov, S., Antonenko, N. (2017). A method for localizing a reference object in a current image with several bright objects. Eastern-European Journal of Enterprise Technologies, 3 (9 (87)), 68-74. doi: https://doi.org/ 10.15587/1729-4061.2017.101920

3. Fursov, V. A., Bibikov, S. A., Yakimov, P. Yu. (2013). Localization of objects contours with different scales in images using Hough transform. Computer Optics, 37 (4), 496-502. doi: https://doi.org/10.18287/0134-2452-2013-37-4-496-502

4. Potapov, A. A. (2013). Fractal paradigm and fractal-scaling methods in fundamentally new dynamic fractal signal detectors. 2013 International Kharkov Symposium on Physics and Engineering of Microwaves, Millimeter and Submillimeter Waves. doi: https:// doi.org/10.1109/msmw.2013.6622151

5. Vasilyeva, I. K., Popov, A. V. (2017). Selection of recognition objects' outer contours on multichannel images. Radioelektronni i kompiuterni systemy, 2 (82), 17-23.

6. Gnilitskii, V. V., Insarov, V. V., Chernyavskii, A. S. (2010). Decision making algorithms in the problem of object selection in images of ground scenes. Journal of Computer and Systems Sciences International, 49 (6), 972-980. doi: https://doi.org/10.1134/ s1064230710060158

7. Mukhina, M. P., Seden, I. V. (2014). Analysis of modern correlation extreme navigation systems. Electronics and Control Systems, 1 (39). doi: https://doi.org/10.18372/1990-5548.39.7343

8. Muñoz, X., Freixenet, J., Cufí, X., Martí, J. (2003). Strategies for image segmentation combining region and boundary information. Pattern Recognition Letters, 24 (1-3), 375-392. doi: https://doi.org/10.1016/s0167-8655(02)00262-3

9. Acevo-Herrera, R., Aguasca, A., Bosch-Lluis, X., Camps, A., Martínez-Fernández, J., Sánchez-Martín, N., Pérez-Gutiérrez, C. (2010). Design and First Results of an UAV-Borne L-Band Radiometer for Multiple Monitoring Purposes. Remote Sensing, 2 (7), 1662-1679. doi: https://doi.org/10.3390/rs2071662

10. Hruska, R., Mitchell, J., Anderson, M., Glenn, N. F. (2012). Radiometric and Geometric Analysis of Hyperspectral Imagery Acquired from an Unmanned Aerial Vehicle. Remote Sensing, 4 (9), 2736-2752. doi: https://doi.org/10.3390/rs4092736

11. Tarshyn, V. A., Sotnikov, A. M., Sydorenko, R. G., Megelbey, V. V. (2015). Preparation of reference patterns for high-fidelity correlation-extreme navigation systems on basis of forming of paul fractal dimensions. Systems of Arms and Military Equipment, 2 (42), 142144.

12. Tarshyn, V. A., Sotnykov, A. M., Sydorenko, R. H. (2015). Preparation of reference patterns for high-fidelity cross-correlationextreme systems of navigation on basis of the use direct cross-correlation analysis. Science and Technology of the Air Force of Ukraine, 2 (19), 69-73.

13. Tarshyn, V. A., Sotnykov, A. M., Sydorenko, R. H. (2014). There are formation principles of complex images reference for precision correlation-extreme navigation systems. Information Processing Systems, 6 (122), 86-89.

14. Fernandes, L. A. F., Oliveira, M. M. (2008). Real-time line detection through an improved Hough transform voting scheme. Pattern Recognition, 41 (1), 299-314. doi: https://doi.org/10.1016/j.patcog.2007.04.003

15. Yeromina, N., Petrov, S., Tantsiura, A., Iasechko, M., Larin, V. (2018). Formation of reference images and decision function in radiometric correlationextremal navigation systems. Eastern-European Journal of Enterprise Technologies, 4 (9 (94)), 27-35. doi: https://doi.org/10.15587/1729-4061.2018.139723

16. Minenko, D. E., Abdirasul, U. T., Ubaychin, A. V. (2018). Effektivnost' primeneniya mikrovolnovyh radiometrov razlichnyh tipov v sostave bortovyh issledovatel'skih sistem malogabaritnyh bespilotnyh letatel'nyh apparatov. Sbornik izbrannyh statey nauchnoy sessii TUSUR, 1-1, 35-40. Available at: https://www.elibrary.ru/item.asp?id=36415317

17. Pavlykov, V. V. (2013). Improvement of primary radiometric images quality in scanning radiometers of compensation type. Information Processing Systems, 4 (111), 27-32.

18. Zhila, S. S. (2014). Optimal'niy algoritm obrabotki signalov v SVCh radiometre s nestabil'nym koeffitsientom usileniya priemnika. Radiotehnika, 177, 77-85.

19. Hersman, M. S., Poe, G. A. (1981). Sensitivity of the Total Power Radiometer with Periodic Absolute Calibration. IEEE Transactions on Microwave Theory and Techniques, 29 (1), 32-40. doi: https://doi.org/10.1109/tmtt.1981.1130283

20. Gol'denberg, L. M., Levchuk, YU. P., Polyak, M. N. (1974). Tsifrovye fil'try. Moscow: Svyaz', 160.

21. Gol'denberg, L. I., Matyushkin, B. D., Polyak, M. N. (1985). Tsifrovaya obrabotka signalov. Moscow: Radio i svyaz', 312.

22. Vereshkin, A. E., Katkovnik, V. Ya. (1973). Lineynye tsifrovye fil'try i metody ih realizatsii. Moscow: Sovetskoe radio, 152.

23. Hurgin, Ya. I., Yakovlev, V. P. (1962). Metody teorii tselyh funktsiy v radiofizike, teorii svyazi i optike. Moscow: GIFML, 220.

24. Gradshteyn, I. S., Ryzhik, I. M. (1962). Tablitsy integralov, summ, ryadov i proizvedeniy. Moscow: GIFML, 1100.

25. Alekseev, V. M., Tihomirov, V. M., Fomin, S. V. (1979). Optimal'noe upravlenie. Moscow: Nauka, 432. 